Article

\title{
High-Capacity Data-Hiding Scheme on Synthesized Pitches Using Amplitude Enhancement-A New Vision of Non-Blind Audio Steganography
}

\author{
Hung-Jr. Shiu ${ }^{1}$, Bor-Shing Lin ${ }^{2}$, Chia-Wei Cheng ${ }^{3}$, Chien-Hung Huang ${ }^{4, *}$ and Chin-Laung Lei ${ }^{1}$ \\ 1 DCNS Lab, Graduate Institute of Electrical Engineering, National Taiwan University, Taipei 10617, Taiwan; \\ hsuhongzhi@gmail.com (H.-J.S.); lei@cc.ee.ntu.edu.tw (C.-L.L.) \\ 2 Department of Computer Science and Information Engineering, National Taipei University, \\ New Taipei City 23741, Taiwan; bslin@mail.ntpu.edu.tw \\ 3 Department of Computer Science and Information Engineering, National Chinan University, \\ Nantou County 54561, Taiwan; cwcheng@gmail.com \\ 4 Department of Computer Science and Information Engineering, National Formosa University, \\ Yunlin County 63201, Taiwan \\ * Correspondence: chhuang@nfu.edu.tw; Tel.: +886-5-631-5588
}

Academic Editor: Vladimir Shpilrain

Received: 30 April 2017; Accepted: 14 June 2017; Published: 17 June 2017

\begin{abstract}
This work proposes a new and non-blind steganographic scheme for synthesized pitches. Synthesized music is popularly used to demonstrate early versions of compositions conveniently and at low-cost. They can also be utilized to pass secrets or obtain digital rights. The method consists of two procedures, of which the first is the realistic simulation of synthesized pitches using a computer and the second is the hiding of secrets during the generated simulated pitches. The first part of this paper reviews attempts to discover the fundamental patterns of synthesized pitches and to develop a strategy for generating approximate pitches using a computer. The component frequencies are used to generate a pitch in which to hide secrets. Legal receivers receive the referenced composition and frequencies, enabling them to generate the synthesized pitches according to the main frequencies of the referenced composition. Finally, the generated and received pitches are compared to identify the secret bits. As more frequencies are used to hide secret bits, more secret bits can be embedded in the synthesized pitches. The use of more frequencies makes synthesized pitches more realistic compared to real ones. The performance of the proposed method is also compared with that of competing methods and under common attacks.
\end{abstract}

Keywords: steganography; data-hiding; synthesized music; amplitude enhancement

\section{Introduction and Related Work}

The Internet is a popular environment in which people exchange personal data. Accordingly, great importance is now attached to information security. Many techniques for keeping data confidential have been developed. The field of steganography concerns hiding data that embed messages in insignificant media before a transmission. Data-hiding schemes are used to hide secrets in cover media, producing stego-media. The approach enables users to discover attempts by intruders to replace original messages with fabricated content. One of its applications is to back-up personal, private data or information on the Internet. Presently, many people frequently upload personal and secret data to cloud services, while reasonably distrusting cloud vendors. The objective of data-hiding is to increase hiding capacity while reducing the likelihood that intruders can identify anything is hidden [1]. Figure 1 displays some applications of steganography: (a) when a user backs up sensitive data on a cloud storage service, he does not want the data accessible even though it is encrypted; 
(b) when a user create his multimedia files, he wants to obtain his ownerships before he publishes those files; and (c) when a user wants to communicate to some, he does not want to be noticed so he makes his messages to be un-perceptual.

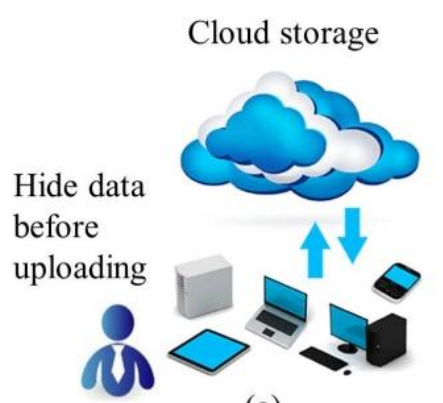

(a)
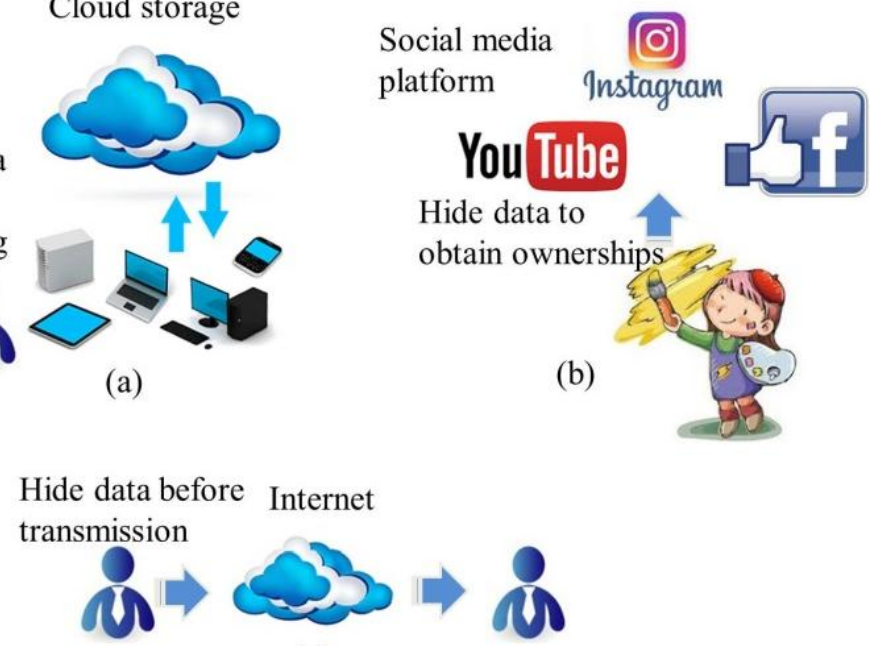

(c)

Figure 1. (a) An application of a user backing up his sensitive data on a cloud storage service; (b) an application of a user making digital rights; and (c) an application of a user achieving an un-perceptual communication.

Popular multimedia includes images, audio, video and text [1-16]. Audio-based data-hiding methods [3,17-23] can be divided into two categories. Time domain-based operations tend to replace the least significant bits $[3,20]$, and the echo parts of a signal [3,23]. Frequency domain-based operations include directly hiding secrets in high or low frequencies and spreading the secrets throughout band frequencies. The former approach is known as psychoacoustic masking [3,21] and the latter is known as spreading the spectrum $[3,22,23]$. Most audio-based methods suffer from the same problems as image-based methods, that is, the issue of distortion between cover and stego-medium. Phase coding and spreading spectrum methods are safer due to they are designed to deployed secrets in un-perceptual frequencies [3], although their computation time are greater than those of other schemes.

Traditional data-hiding strategies are based on digitalized multimedia. Distortion is the most important limitation of steganography, and it must therefore be controlled to reduce awareness of the generated stego-media. Other methods for hiding data in audio are based on ideas that have been proposed by various authors [3,20-23]. They include least-significant-bit (LSB), phase coding, spread spectrum, echo data-hiding and psychoacoustic masking methods. Akhaee et al. [17] proposed a robust data-hiding algorithm to avoid statistical cracking. They found that most time-domain processing data-hiding schemes are weak and can be easily decoded by current steganalytic strategies. Their algorithm applies correlated quantization to embed data using a histogram-based detector. Huang et al. [19] presented a new steganographic scheme with variable capacity and synchronization for the secure multimedia transmission of acoustic data in real time. Atoum et al. [18] proposed a new data-hiding scheme that is based on the mp3 file format. They were concerned that human ears are very sensitive to audio features so the audio content should not be modified. Their method, therefore, hides secret data only between the frames of an mp3 file. Yamamoto and Iwakiri [24] developed a method of identifying the ownership of a digital instrumental audio. They mentioned that digital instrumental audio is now very popular on the Internet. Accordingly, the present work develops a data-hiding scheme that is based on the fundamental composition of simulated instrumental audio.

Most of the above proposed methods are based on traditional digitalized audio and are therefore restricted by distortion and exhaustion of transmission, which means the more secrets embedded in a 
cover media, the more differences of a cover and a stego media. They can be used simply to modify media content, but in so doing, they limit the capacity for and security of the hidden secrets. Currently, much content is transmitted through the Internet, and a fraction of it is made by computer animators, and includes text and simulated media [25-32]. Cayre and Macq [28] were the first to propose a data-hiding method that was based on 3D polygons. Their scheme is applied to 3D meshes of triangles, and extends one of the simplest data-hiding techniques, called the triangle strip peeling sequence (TSPS) technique. The basic idea of the TSPS algorithm is the insertion of bits in a path traced on the mesh. In 2009, Chao et al. [29] improved Cayre's algorithm and increased its capacity. They redefined 3D polygons as multilayered triangles such that each layer could be used to hide secrets. They also used coordinates to define each polygon and defined a multi-layer such that the capacity of their method was $3 n$ times that of Cayre's method (where $n$ is the number of layers).

Inspired by the above research works, the authors developed a new data-hiding scheme that uses synthesized musical pitches to embed more secrets and reduce distortion. The scheme reproduces a synthesized musical pitch in which it embeds secrets by amplitude enhancement. The enhancement slightly pads noises and legal receivers have only to compare the magnitude of the amplitude with that in standard patterns of synthesized pitches. The restriction is that only one pitch can be used at the same time. Sound synthesis is an important topic in the simulation of digital musical instruments. This paper is organized as follows. Section 2 first introduces the fundamental principle of the synthesis of musical notes, which is the basis of the data-hiding scheme that is presented herein. A reliable formula for synthesizing notes is introduced and some simulations are carried out. Therefore, a data-hiding scheme are presented. Section 3 displays some practical experiments to prove the feasibility and ability. Section 4 compares the performance of the proposed method with of others and presents some other evaluations and theoretical analysis. Finally, Section 5 draws conclusions. All experiments are analyzed using MATLAB (2006a, The MathWorks, Natick, MA, USA).

\section{Materials and Methods}

References [33,34] described efforts to describe objectively the quality of piano tones, as understood by musicians, and they tried to find synthetic tones that would be considered to be better than real piano tones. Casey showed that a two-layer feed-forward model can perform inverse mapping for a simple physical model of a string [35]. References [36,37] showed the numerical approach and the underlying physical model can be improved to simulate the motion of a piano string with a high degree of realism. This work develops a model of instruments as follows. First, a discrete Fourier transform (DFT) is utilized to transform the sampled sound data of an instrument from the time domain to the frequency domain. When a real instrumental pitch is recorded, analog acoustic is digitalized with being sampled automatically by a computer. Then the DFT could be adopted to classify and decompose the composition of the frequencies of a single pitch. Second, use the frequency domain function and the sound of an instrument is described as a pattern, which is generated using DFT and the inverse DFT, which are used to analyze sampled data using computers. Section 2.1 is the fundamental of the methodology, while Section 2.2 is the proposed scheme.

\subsection{Fundamentals}

This section discusses why musical instruments produce such beautiful music. Harmonics will be introduced. Fourier transformations are based on the fact that a function in the time domain can be represented as a summation of cosine functions. Consider the periodic square wave, plotted in Figure 2. The signal $x(t)$ can be represented by Equation (1) [38]:

$$
x(t)=\frac{2 T_{s}}{T}+\sum_{k=1}^{\infty} \frac{2 \sin \left(2 \pi k f_{0} T_{s}\right)}{\pi k} \cos \left(2 \pi k f_{0} t\right)
$$




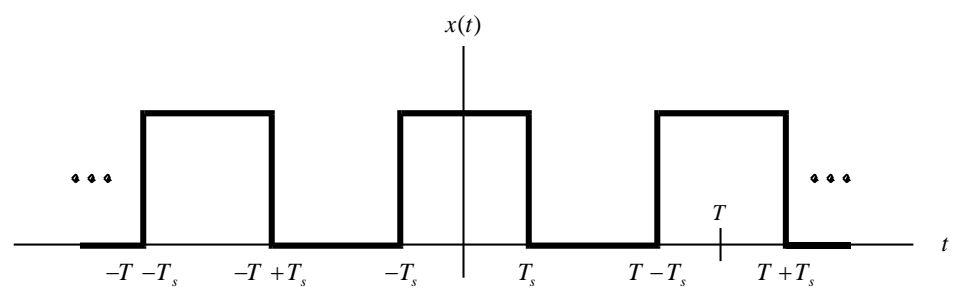

Figure 2. A square wave signal [38].

The frequency $f_{0}$ is the fundamental frequency. As $k$ increases, the coefficient of the cosine function, $\frac{2 \sin \left(2 \pi k f_{0} T_{s}\right)}{\pi k}$, decreases. Hence, only the coefficients for $k=2,3,4$ and other low values are important. The cosine function for $k=2$ is known as the second harmonic; that for $k=3$ is the third harmonic, and so on. The sound of any musical note that is produced by a musical instrument contains the fundamental frequency and a few harmonics. Figure 3 plots the function of a real piano's Middle $C$ in the time domain. The DFT is applied to the function in Figure 3 to obtain the frequency spectrum in Figure 4. Only a few of the magnitudes are marked because space is limited. After the frequency spectrum of Middle $C$ on a real piano was obtained, the frequency spectra of all of the pitches that are produced in the middle region of a piano are found. Figure 5 displays these spectra.

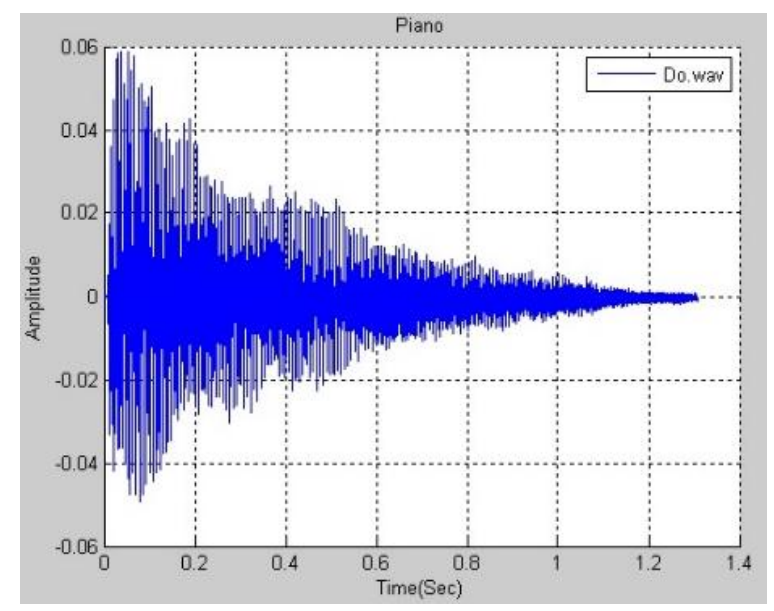

Figure 3. The time domain function of a real piano's Middle C.

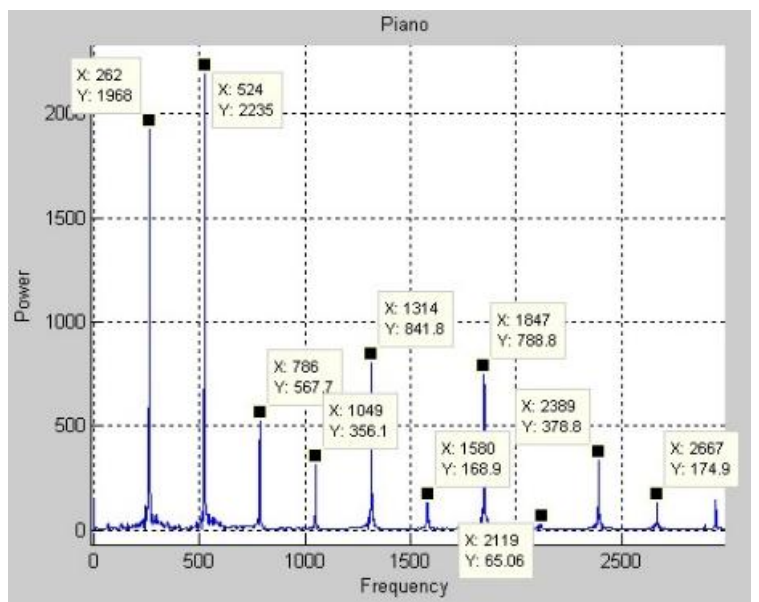

Figure 4. Discrete Fourier Transform (DFT) of real piano Middle C. 


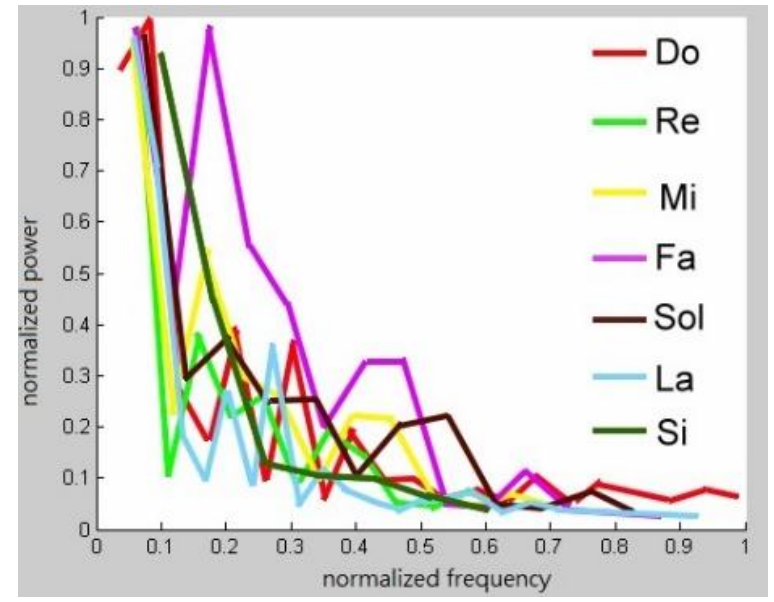

Figure 5. Frequency spectra of all piano pitches.

A real music instrument produces different pitches with different frequency patterns. Consider a randomly chosen musical instrument, such as a piano and the playing of any note on it. Perform a DFT on the note. The $k$ frequencies with the largest magnitudes are selected. Denote these frequencies as $f_{1}, f_{2}, \ldots, f_{k}$, where $f_{1}<f_{2}<\ldots<f_{k}$, with magnitudes $a_{1}, a_{2}, \ldots, a_{k}, 0 \leq a_{i} \leq 1$. Calculate $b_{i}=f_{i} / f_{1}$ for $1 \leq i \leq k$. Now suppose that the goal is to generate a frequency pattern for Middle $C$. The fundamental frequency of Middle $C$ is known to be $262 \mathrm{~Hz}$. Denote this frequency as $f_{a p}$. The Middle $C$ that is generated by a real piano has frequencies $b_{1} f_{a p}, b_{2} f_{a p}, \ldots, b_{k} f_{a p}$ with magnitudes $a_{1}, a_{2}, \ldots, a_{k}$, respectively, and the inverse DFT generates the sound from these frequencies. Of course, musical sounds that are generated in this way are not expected to be the same as those produced by a piano. However, as demonstrated by the following experiment, they will be piano-like if $k$ is sufficiently large. The $k$ frequencies with the largest magnitudes are selected using the prune and search method [39].

The following experiments involve synthesized pitches. Let $k=10$ so the ten frequencies with the largest magnitudes are obtained. The magnitudes $a_{1}, a_{2}, \ldots, a_{10}$ and respective multiples $b_{1}, b_{2}, \ldots, b_{10}$ are found and shown in Table 1. Middle $C$ on a piano has frequencies $\left(f_{a p}, 1.0038 f_{a p}, \ldots, 7.0843 f_{a p}\right)$ with respect magnitudes $(0.2635,0.7042, \ldots, 0.2402)$. Let $f_{a p}=262$, yielding frequencies of $(262$, $263, \ldots, 1856 \mathrm{~Hz}$ ). Figures 6 and 7 plot the experimental results in the frequency and time domains. Comparing Figures 3 and 7, the waves are not similar.

Table 1. The values of $a_{i}, b_{i}$ and $b_{i} f_{a p}$ of Middle $C$ of a piano with $k=10$.

\begin{tabular}{cccc}
\hline $\boldsymbol{i}$ & $\boldsymbol{a}_{\boldsymbol{i}}$ & $\boldsymbol{b}_{\boldsymbol{i}}$ & $\boldsymbol{b}_{i} f_{\boldsymbol{a} \boldsymbol{p}}$ \\
\hline 1 & 0.2635 & 1.0000 & 262 \\
2 & 0.7042 & 1.0038 & 263 \\
3 & 0.5050 & 1.0077 & 264 \\
4 & 0.3326 & 2.0038 & 525 \\
5 & 0.8000 & 2.0077 & 526 \\
6 & 0.2255 & 2.0115 & 527 \\
7 & 0.3013 & 5.0345 & 1320 \\
8 & 0.2823 & 7.0766 & 1854 \\
9 & 0.2631 & 7.0805 & 1855 \\
10 & 0.2402 & 7.0843 & 1856 \\
\hline
\end{tabular}




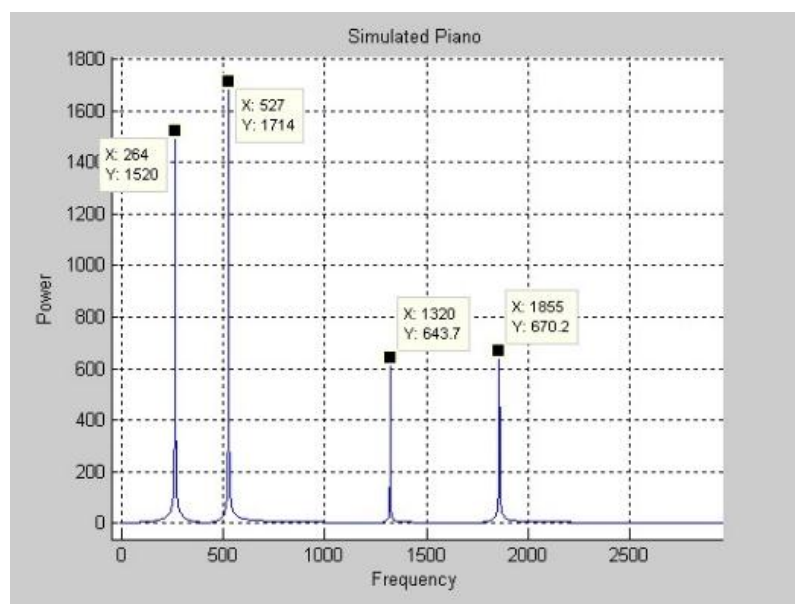

Figure 6. The frequency domain with $k=10$.

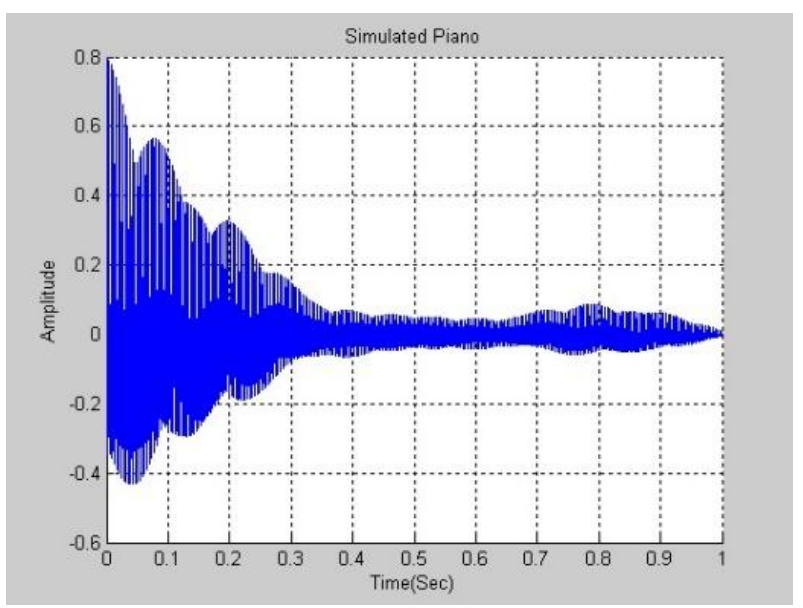

Figure 7. The time domain function of Middle $\mathrm{C}$ of a simulated piano with $k=10$.

Figure 8 presents the similarity between a simulated piano note with $k=10,000$ and a real piano note. The difference between them is negligible and this fact will be exploited in the following section. A higher $k$ yields a smaller distortion and the higher ability to embed more secrets. Various pitches from different musical instruments were simulated and analyzed.
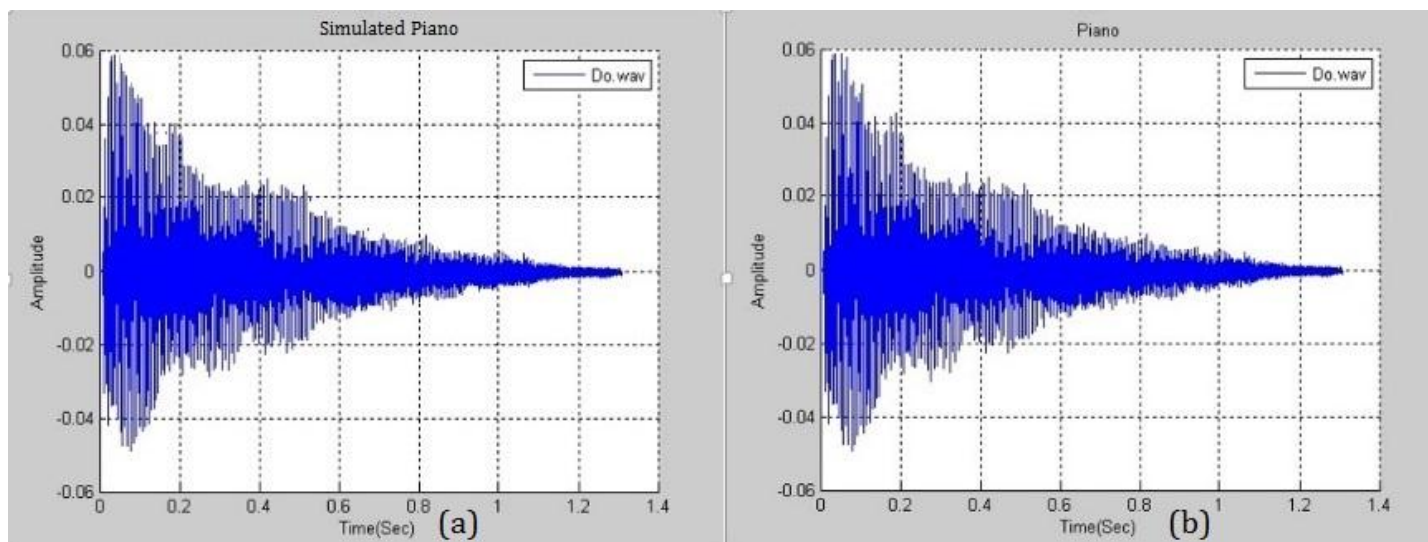

Figure 8. The time-domain comparison between simulated piano with: (a) $k=10,000$; and (b) real piano. 


\subsection{Data Hiding Scheme}

The proposed data-hiding scheme involves the following steps. First, choose an instrumental pitch $P$ to be the reference pitch. With reference to the preceding sections, all of the required parameters can be obtained. These include the magnitudes $a_{i}$ and the main frequencies $b_{i} f_{a p}$. The number of $a_{i}$ terms is $k$ and the number of $b_{i} f_{a p}$ terms is $k$ because the system is used only to generate the main $k$ frequencies of the signals. Next, suppose that the length of the secret bit stream $b t_{1}, b t_{2}, \ldots, b t_{k}$ is also $k$. If $b t_{1}=1$, then $a_{1}$ is increased to $\sigma a_{1}, 1<\sigma<2$; the same operation is applied to $a_{2}, a_{3}, \ldots, a_{k}$. Figure 9 is the overview of the scheme. The sender uses the standard pattern of synthesized pitches and $k$ to encode the secrets to generate a synthesized pitch using amplitude enhancement. A legal receiver uses the standard pattern of synthesized pitches and $k$ to decode the secret.

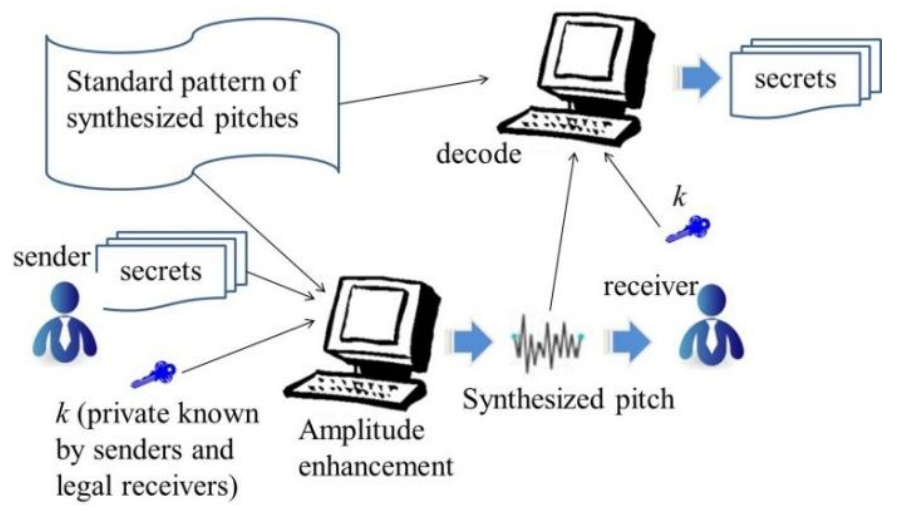

Figure 9. An overview of the proposed scheme.

Algorithm 1 is the simple encoding procedure. Algorithm 2 is the decoding procedure.
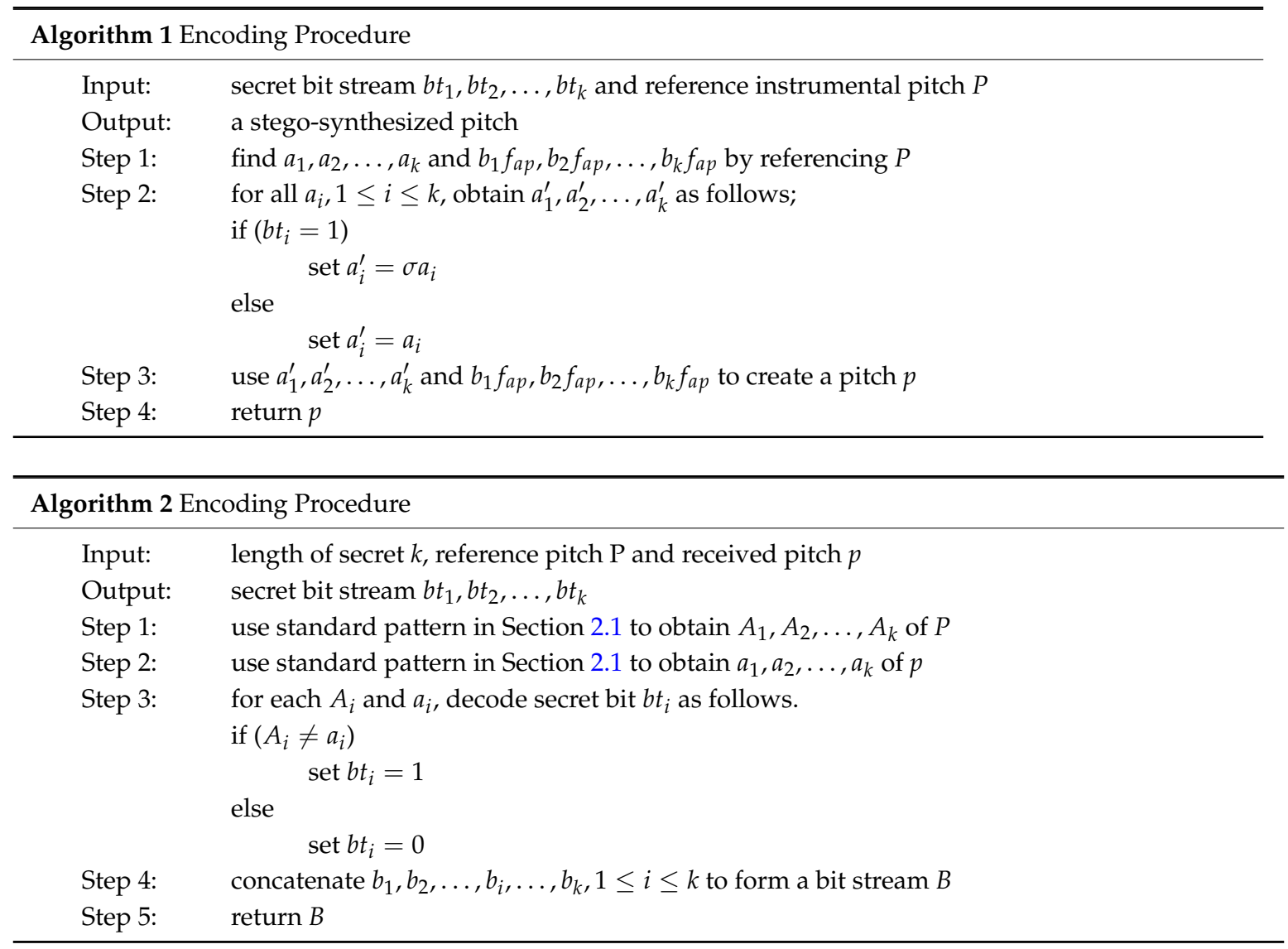
The value $(\sigma-1) a_{1}$ shall be too small to be perceived by the human ear. Consider, for example, Table 2: hiding bit stream 1001101101 in the synthesized pitch yields the enhanced magnitudes, which are shown in the first table.

Table 2. The modified parameters after embedding secret 1001101101 in Middle $\mathrm{C}$ of a simulated piano with $\sigma=1.01$.

\begin{tabular}{cccc}
\hline $\boldsymbol{i}$ & $\boldsymbol{a}_{\boldsymbol{i}}$ & $\boldsymbol{b}_{\boldsymbol{i}}$ & $\boldsymbol{b}_{\boldsymbol{i}} f_{\boldsymbol{a} \boldsymbol{p}}$ \\
\hline 1 & 0.2661 & 1.0000 & 262 \\
2 & 0.7042 & 1.0038 & 263 \\
3 & 0.5050 & 1.0077 & 264 \\
4 & 0.3359 & 2.0038 & 525 \\
5 & 0.8080 & 2.0077 & 526 \\
6 & 0.2255 & 2.0115 & 527 \\
7 & 0.3043 & 5.0345 & 1319 \\
8 & 0.2851 & 7.0766 & 1854 \\
9 & 0.2631 & 7.0805 & 1855 \\
10 & 0.2426 & 7.0843 & 1856 \\
\hline
\end{tabular}

Legitimate receivers obtain the reference instrumental pitch $P$, the length of the secret $k$ and the method of generation of the synthesized pitches. The decoding procedure is as follows. First, identify the main frequencies that correspond to the $k$ largest magnitudes from $P$ and the received pitch $p$. The frequencies of the former are denoted as $F_{1}, F_{2}, \ldots, F_{k}$ and those of the latter are denoted as $f_{1}, f_{2}, \ldots, f_{k}$. The magnitudes of all main frequencies are obtained as $A_{1}, A_{2}, \ldots, A_{k}$ and $a_{1}, a_{2}, \ldots, a_{k}$. Each $A_{i}$ is compared with the corresponding $a_{i}$; for Algorithm 4, Step 3, if $A_{i} \neq a_{i}$, then the secret bit $b t_{i}=1$; otherwise, $b t_{i}=0$. Finally, all $b t_{i}$ s are concatenated and the secret bit stream can be produced.

While the synthesis of musical pitches and the data-hiding scheme are public, the above algorithms can be designed more secure by including a parameter $R$, which is the real order of $a_{i}$ and $b_{i} f_{a p}$ during the data embedding procedure. $R$ is generated using a random number generator and the seed of the generator is obtained by legal receivers. The formal definition of $R$ is $R=\left\{r_{i}\right\}, 1 \leq r_{i} \leq k$, where all $r_{i}$ have different values. An example follows. Consider $R=\{3,1,7,9,2,10,4,6,5,8\}$; Table 3 is obtained after the complex version of the data embedding scheme is implemented. The red numbers indicate hidden secret "1" bits. Evidently, the positions of the secret bits differ from those in the second table. Algorithms 3 and 4 describe the complex version of the proposed scheme.

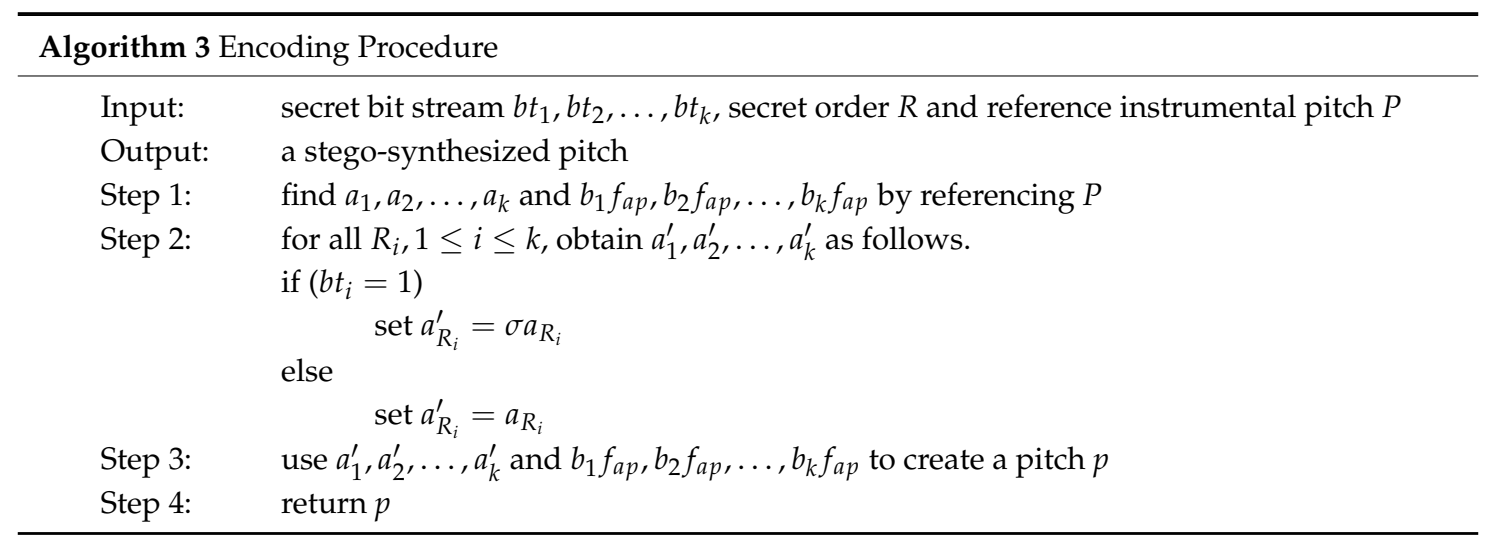


Table 3. The modified parameters after embedding secret 1001101101 in Middle $C$ of a simulated piano with $R=\{3,1,7,9,2,10,4,6,5,8\}$ and $\sigma=1.01$.

\begin{tabular}{cccc}
\hline $\boldsymbol{i}$ & $\boldsymbol{a}_{\boldsymbol{i}}$ & $\boldsymbol{b}_{\boldsymbol{i}}$ & $\boldsymbol{b}_{\boldsymbol{i}} f_{a \boldsymbol{p}}$ \\
\hline 1 & 0.2635 & 1.0000 & 262 \\
2 & 0.7112 & 1.0038 & 263 \\
3 & 0.5101 & 1.0077 & 264 \\
4 & 0.3359 & 2.0038 & 525 \\
5 & 0.8000 & 2.0077 & 526 \\
6 & 0.2278 & 2.0115 & 527 \\
7 & 0.3013 & 5.0345 & 1320 \\
8 & 0.2851 & 7.0766 & 1854 \\
9 & 0.2657 & 7.0805 & 1855 \\
10 & 0.2402 & 7.0843 & 1856 \\
\hline
\end{tabular}

Unlike in the first version, receivers no longer need the value of $k$ but only the secret order $R$ or random seed.

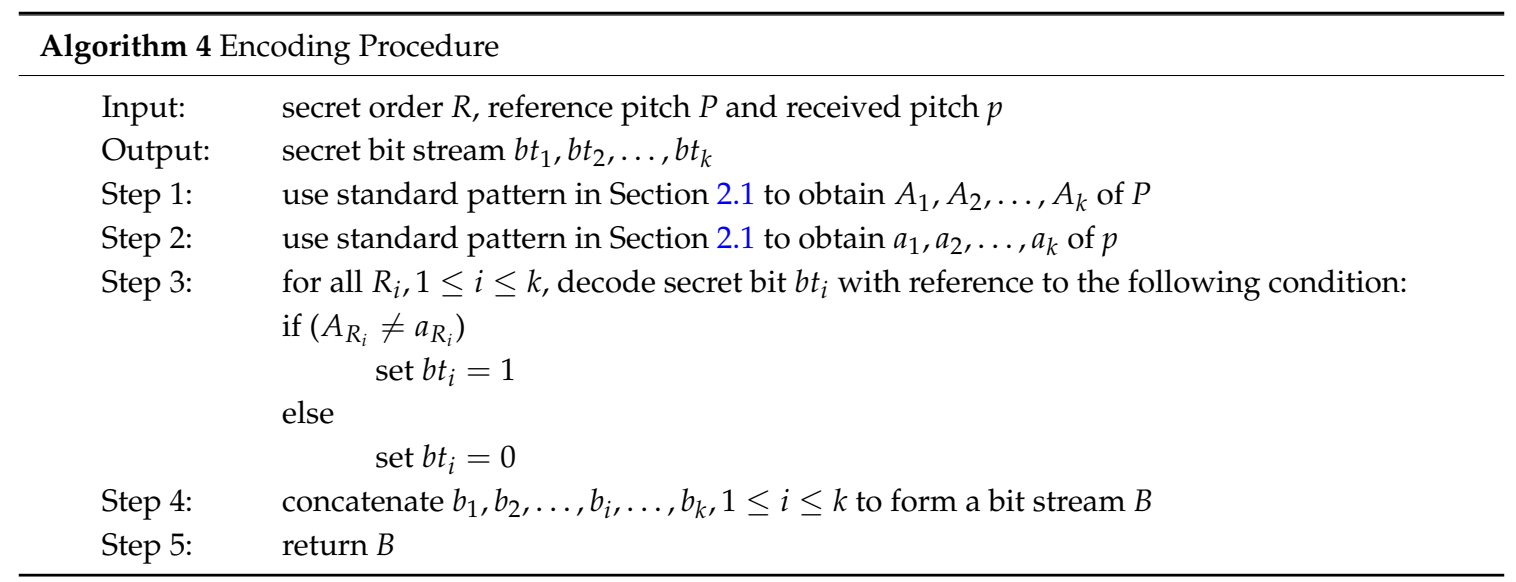

The above two proposed embedding schemes focus on enhancing amplitudes when secret " 1 " bits are embedded. However, it shall be considered that the enhancement will be too large if there are too many " 1 " bits. A strengthened version is presented here called alternating current (AC) algorithm. The main idea of AC algorithm is to reduce a large enhancement caused by embedding secret " 1 " bits. The embedding scheme goes on alternatively enhancing each amplitude by multiply $\sigma$ and $\frac{1}{\sigma}$. For the example in Table 3, the parameters are modified as listed in Table 4 by adopting AC algorithm. The numbers of the even positions $(9,4,8)$ of embedding secret " 1 " bits are modified by multiplying $\frac{1}{\sigma}$. Algorithms 5 and 6 describe the embedding and extracting procedures of the AC algorithm, respectively.

Table 4. The modified parameters after embedding secret 1001101101 in Middle $\mathrm{C}$ of a simulated piano using the alternating current (AC) algorithm with $R=\{3,1,7,9,2,10,4,6,5,8\}$ and $\sigma=1.01$.

\begin{tabular}{cccc}
\hline $\mathbf{i}$ & $\boldsymbol{a}_{\boldsymbol{i}}$ & $\boldsymbol{b}_{\boldsymbol{i}}$ & $\boldsymbol{b}_{\boldsymbol{i}} \boldsymbol{f}_{\boldsymbol{a p}}$ \\
\hline 1 & 0.2635 & 1.0000 & 262 \\
2 & 0.7112 & 1.0038 & 263 \\
3 & 0.5101 & 1.0077 & 264 \\
4 & 0.3293 & 2.0038 & 525 \\
5 & 0.8000 & 2.0077 & 526 \\
6 & 0.2278 & 2.0115 & 527 \\
7 & 0.3013 & 5.0345 & 1320 \\
8 & 0.2795 & 7.0766 & 1854 \\
9 & 0.2605 & 7.0805 & 1855 \\
10 & 0.2402 & 7.0843 & 1856 \\
\hline
\end{tabular}



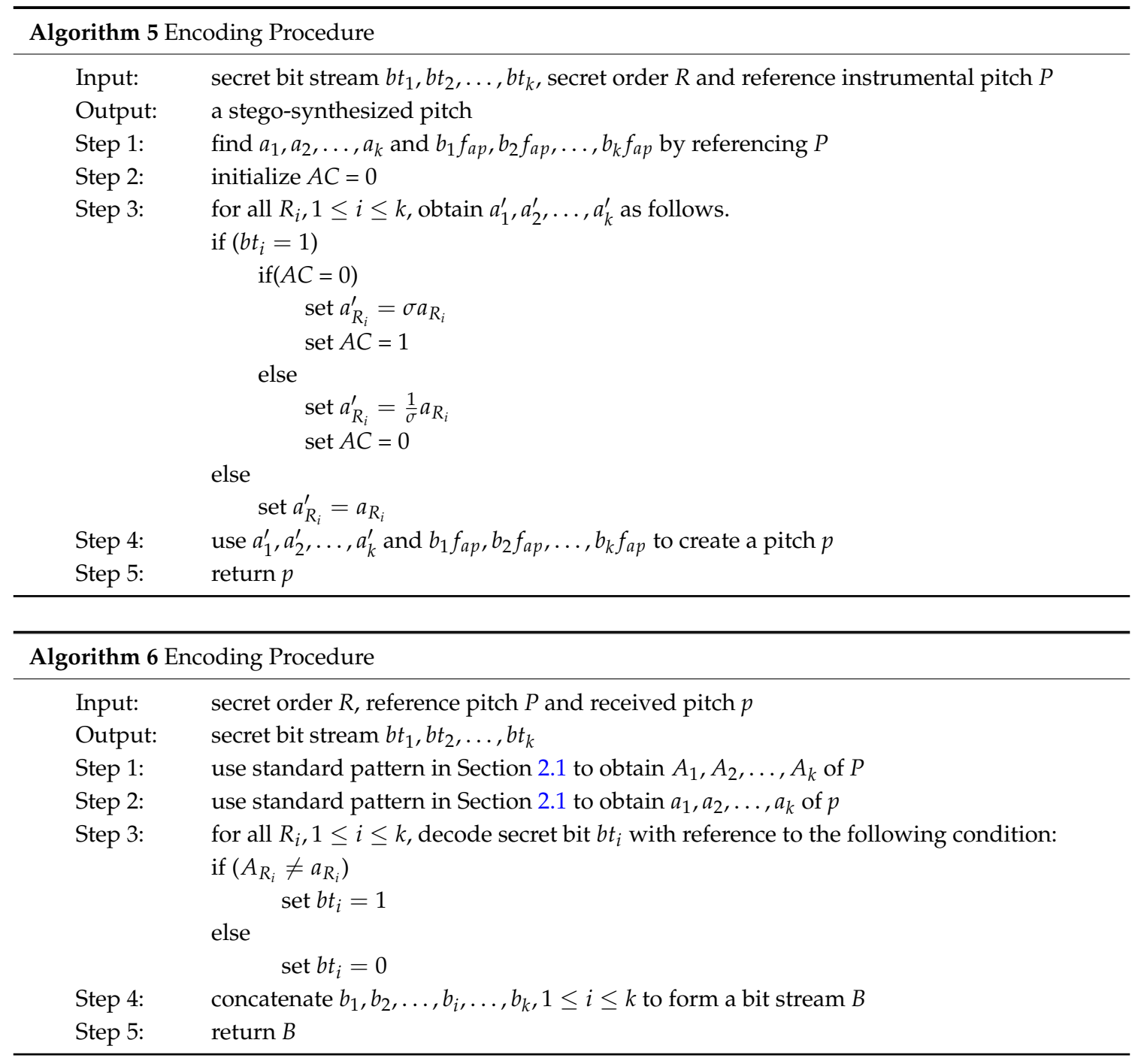

It shall be proven that even if the proposed steganographic scheme is applied twice, it is still able to get the embedded secrets. A simple proof is given as follows. The basic idea of this presented research work is the enhancement of amplitudes if embeds a secret " 1 " bits. Applying the embedding procedure twice makes the enhanced amplitudes multiply $\sigma$ of $\frac{1}{\sigma}$ again, that is, $a_{i}$ will be $\sigma^{2} a_{i}$ or $\frac{1}{\sigma^{2}} a_{i}$ but not $\sigma a_{i}$ or $\frac{1}{\sigma} a_{i}$. When legal receivers apply the decoding procedure, the main strategy is to compare the enhanced amplitudes to the original amplitudes of the standard patterns. The are only three possible values of $a_{i}: a_{i}, \sigma^{2} a_{i}$, and $\frac{1}{\sigma^{2}} a_{i}$. Apparently, the comparisons work and are able to obtain the embedded secrets.

\section{Results}

This section displays experiments of the proposed scheme. Figure 10 displays the cover and the stego-pitches with $k=10$. The red numbers indicate secret " 1 " bits that are hidden. Figure 11 shows that the cover and the stego-pithes when $k=10,000$ by embedding 10,000 random bits and the differences are nearly none because the enhancement of amplitudes is too small. Figure 12 plots the signal-to-noise ratio (SNR) between the embedded noise and the standard pitch as a function of $\sigma$ from 1.001 to 1.01 for $k$ from 10 to 50. A larger $\sigma$ causes greater distortions of the cover pitches.

The performance of a data-hiding scheme is generally measured using capacity and distortion. As mentioned above, more multimedia are being produced by Internet users, so intruders have difficulty in distinguishing stego-media from cover media. This work develops a new cover medium, and comparisons can only be made between frequencies for different values of $k$, as selected by the 
standard pattern in Section 2.1. First, the capacity of the proposed scheme is discussed. Since the proposed scheme embeds a secret bit in a selected pitch, the capacity increases with the number of selected pitches. Figure 13 displays the capacity for different values of $k$; a larger $k$ allows more secret bits to be embedded. The value of $k$ is linearly related to capacity.

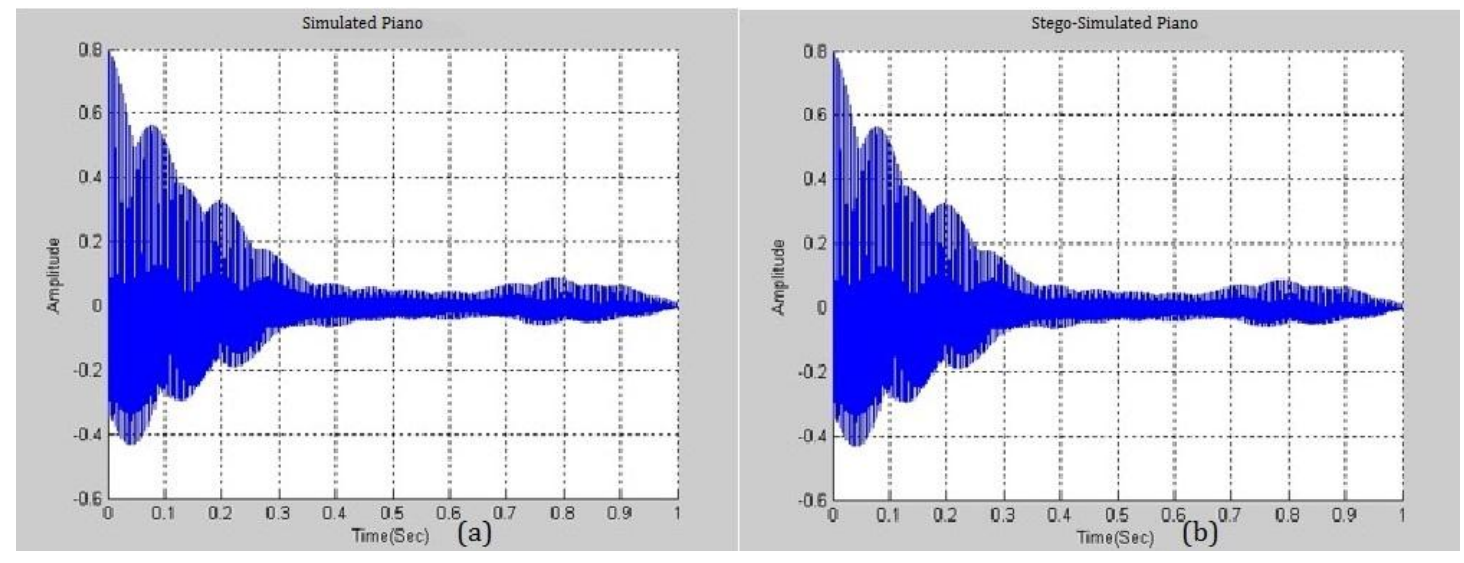

Figure 10. The time-domain comparison between: (a) cover pitch; and (b) stego-pitch with $k=10$ and $\sigma=1.01$

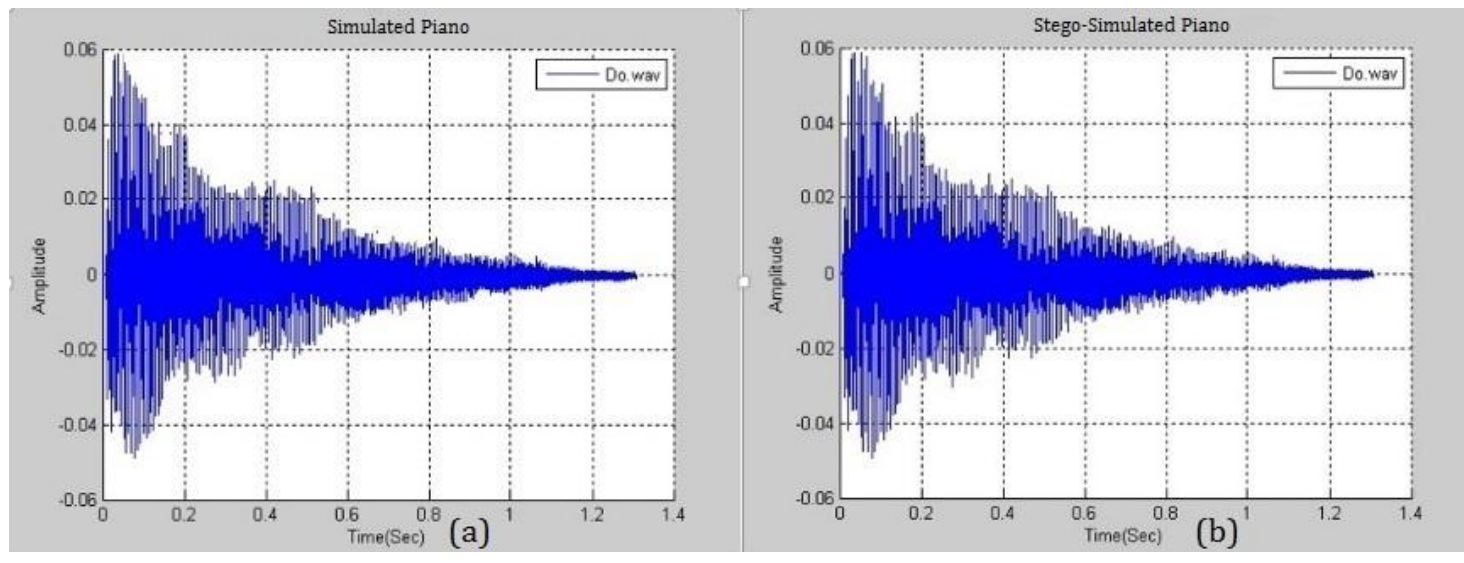

Figure 11. The cover (a); and the stego-pithes (b) when $k=10,000$ and $\sigma=1.01$.

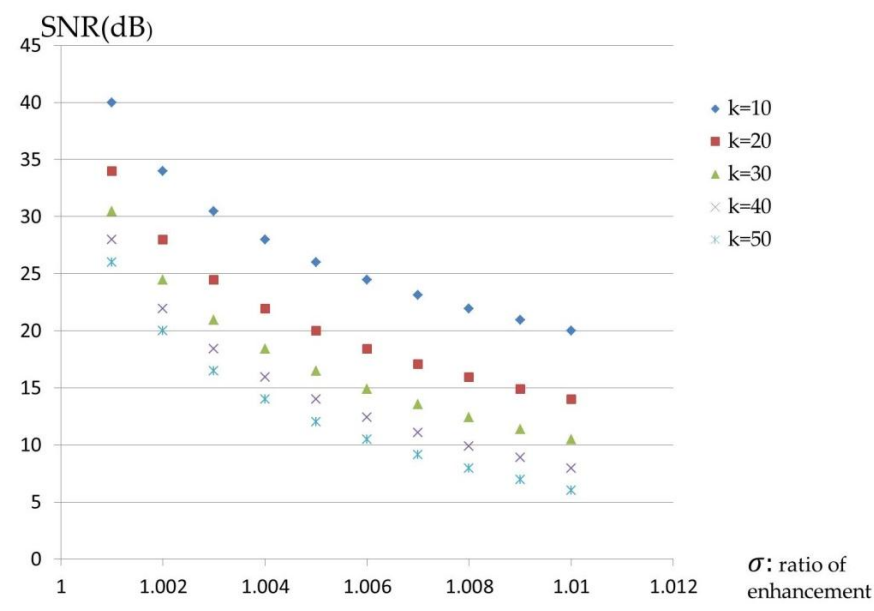

Figure 12. Correlation between different $k$ values and $\sigma$ values. 
Number of embedded bits

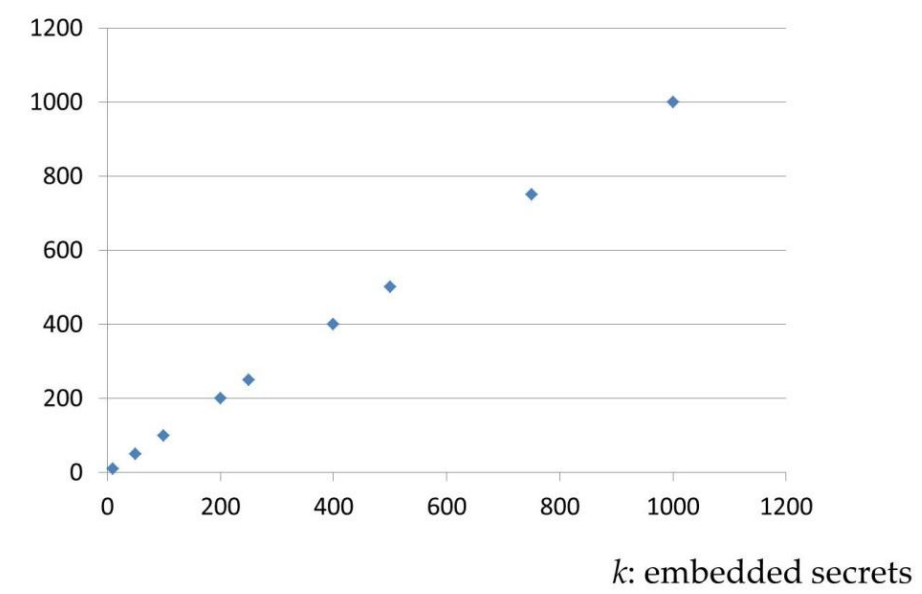

Figure 13. The curvy of capacity to $k$

Second, the distortions between the stego and real pitches are provided for different values of $k$ and $\sigma$. The following experiment is conducted. The peak-signal-to-noise ratio (PSNR) function in the MATLAB library is utilized to evaluate the distortion of two plotted images of stego and real pitches. Figure 14 plots the variation of the PSNR associated with different values of $k$ and $\sigma$. Each point represents a comparison between a stego and a real pitch; for example, the point $(10,000,35)$ compares a stego pitch (with 10,000 bits hidden, $\sigma=1.0001$ ) and a real pitch.

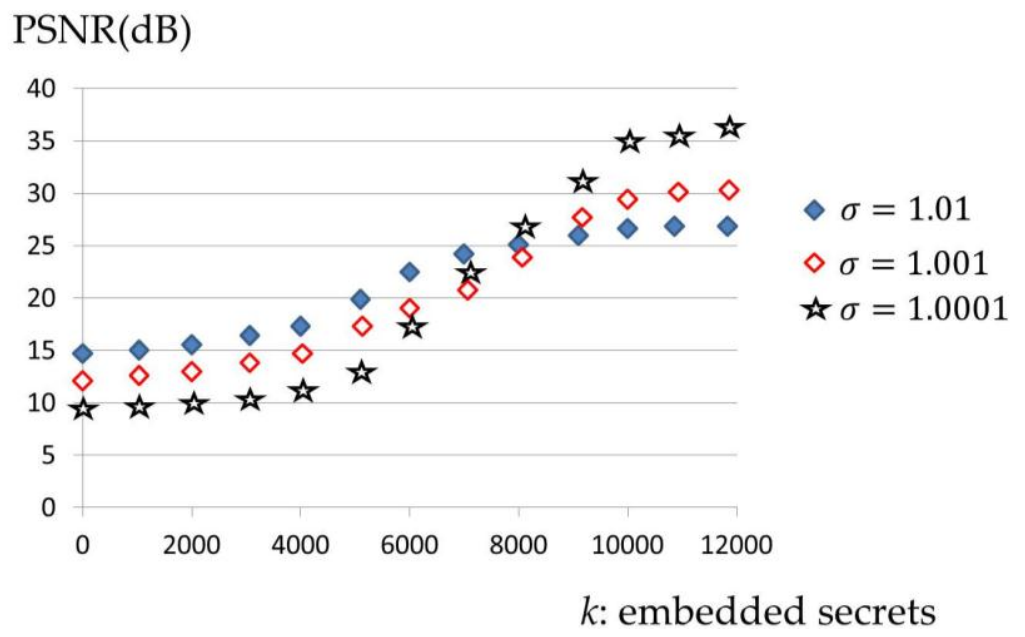

Figure 14. The curve of capacity $(k)$ to distortion (PSNR) between real and simulated stego-pitches with different $\sigma$ values.

The above curve reveals that the proposed scheme yields a smaller distortion as more secret bits are embedded up to a limit beyond which too many secret bits are hidden. In the proposed experiment, the upper bound of the distortion is $37 \mathrm{~dB}$ when $\sigma=0.0001$ and 12,000 bits are embedded. In practical applications, the advantage of the present scheme is that the capacity grows and the distortion declines.

Third, the computational performance of the proposed scheme is addressed. Figure 15 plots the time consumption of the proposed scheme. 


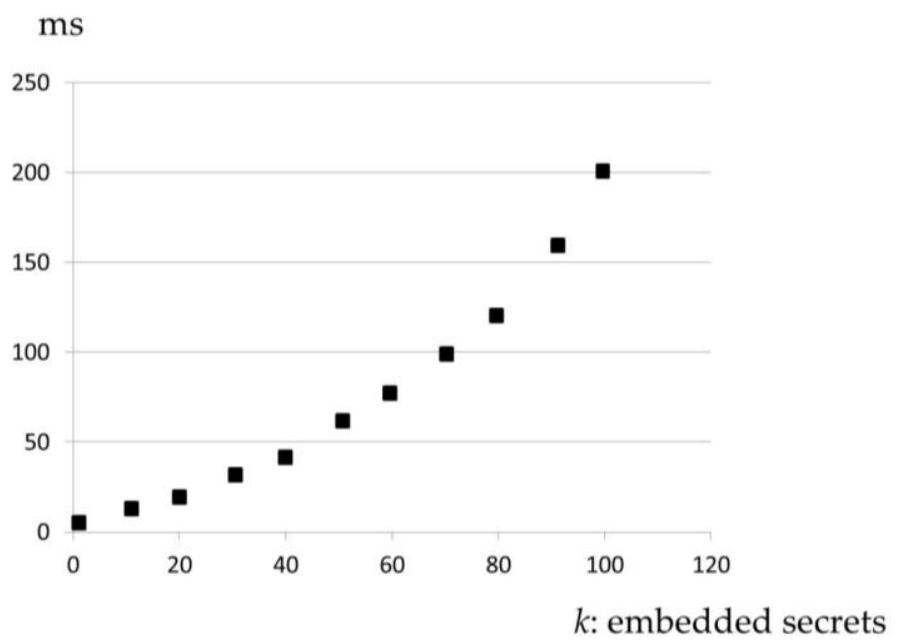

Figure 15. The curve of computational performance to $k$.

\section{Discussion}

This section presents the comparison with other related work, performance under other attacks and some theoretical analysis.

\subsection{Comparisons with Related Work}

Common attacks against an audio data-hiding scheme include the low pass filter (LPF) attack, mp3-like compression, and re-quantization. The bit error ratio $(B E R)$ is used to measure the performance of a data-hiding scheme under common attacks. The BER is defined as the ratio of exact matching of the embedded secret bit-stream and of the decoded secret bit-stream after the stego cover pitch has undergone by the above common attacks. The mathematical definition is:

$$
B E R=\frac{\sum_{i=1}^{k} B_{i}^{\prime} \oplus B_{i}}{|B|}(\oplus \text { is exlusive or })
$$

The definitions of the common attacks adopted in the proposed comparisons are as follows:

- $\quad$ LPF $(3 \mathrm{kHz})$ filters all signals with frequencies lower than $3 \mathrm{kHz}$.

- $\quad$ mp3 (64 kbps) adopts an existing multimedia tool (Adobe Audition) to compress the stego-pitch (.wav file->.mp3 file) and decompress back to .wav file format.

- Re-quantization (16 to 32 bits) adopts an existing multimedia tool (Adobe Audition) to re-quantize the sampling point from 16 bits to 32 bits and then to re-quantize it back to 16 bits.

- Re-quantization (16 to 8 bits) adopts an existing multimedia tool (Adobe Audition) to re-quantize the sampling point from 16 bits to 8 bits and then to re-quantize it back to 8 bits.

Table 5 compares the BERs of the proposed scheme and methods proposed elsewhere $[17,40,41]$. The results indicate that the methods developed herein outperform the others under the indicated attacks. In [17], the authors proposed two steganographic methods, hard quantization (HQ) and soft quantization (SQ) using correlated quantization to embed data with histogram based detector. A novel mapping denoted as point to point graph (PPG) is used to evaluate the correlation among each value of samples. PPG point radii are suggested to embed data to obtain the performances. In [40], the authors presented a self-synchronization scheme for audio watermarking. The synchronization codes are hidden into audio as the informative data, thus the embedded data have the ability of self-synchronization. The synchronization codes are hidden into low frequency coefficients in discrete wavelet transform domain. In [41], the authors proposed an echo hiding scheme. Some echoes are adequately adapted when the embedding process is executing. 
The proposed scheme protects all of the hidden secrets under the specified common attacks for the following reasons.

Table 5. The bit error ratio (BER) of the proposed methods and related work. LPF: low pass filter; HQ: hard quantization; SQ: soft quantization.

\begin{tabular}{ccccc}
\hline Methods/Attacks & LPF (3 kHz) & mp3 (64 kbps) & $\begin{array}{c}\text { Re-Quantization } \\
\mathbf{1 6}-\mathbf{3 2} \text { bits }\end{array}$ & $\begin{array}{c}\text { Re-Quantization } \\
\mathbf{1 6 - 8} \text { bits }\end{array}$ \\
\hline Ours $(k=40)$ & $0.0 \%$ & $0.0 \%$ & $0.0 \%$ & $0.0 \%$ \\
Akhaee et al. [17]: HQ, SQ & $15.0 \%, 15.0 \%$ & $10.2 \%, 0.1 \%$ & $0.0 \%, 0.0 \%$ & $0.0 \%, 0.0 \%$ \\
Wu et al. [40] & $\varnothing$ & $4.3 \%$ & $\varnothing$ & $\varnothing$ \\
Chen et al. [41] & $\varnothing$ & $6.5 \%$ & $\varnothing$ & $11.9 \%$ \\
\hline
\end{tabular}

$\varnothing$ : Not mentioned by the corresponding authors.

LPF (3 kHz), by the definition of the LPF, frequencies higher than $3 \mathrm{kHz}$ will be eliminated under this attack, while in the proposed scheme, the selected $k$-pitches are all beyond $3 \mathrm{kHz}$ such that no bits are eliminated. mp3 (64 kbps), the main purpose of mp3 compression is to eliminate frequencies higher than $22 \mathrm{kHz}$, and as under the LPF attack, the proposed scheme selects all low frequencies so that no bits disappear. Re-quantization (16 to 32 bits), this attack increases the size of the binary representation of numbers and extension will not affect the sampled values of the stego-pitches. Evidently, no secret bits will be destroyed. Re-quantization (16 to 8 bits), this attack shrinks the size of the binary representation of numbers, eliminating the suffix numbers when is re-quantized to its original size. In the proposed scheme, the selected $\sigma$ does not produce long floating numbers after the enhancement of amplitudes so the scheme performs well under this attack.

Another fact of the comparison is the curve of the trend among capacity and distortion. Figure 16 demonstrates the difference of the curve between the related work [17] and the proposed work. It can be seen that the distortion decreases when the capacity increases, while, in this work, the distortion increases to a boundary value when the capacity increases. The phenomenon is because the proposed scheme is designed for breaking the bottleneck of traditional steganography, as illustrated in Figure 16a.
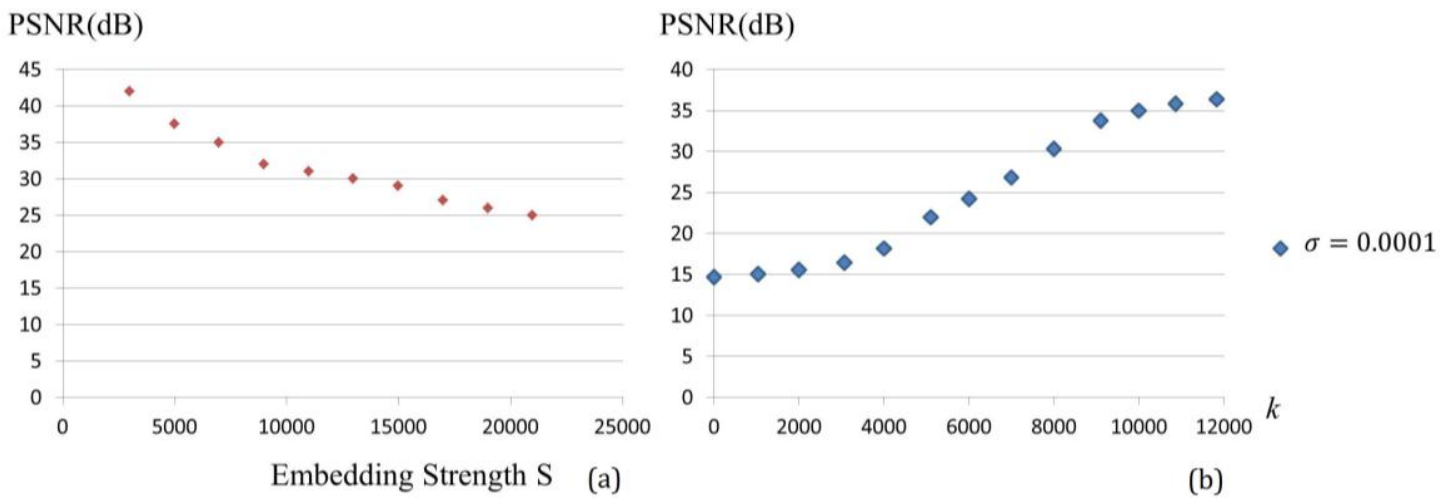

Figure 16. The curve of the trend of distortion and capacity: (a) proposed in [17]; and (b) the work presented by the authors.

\subsection{Performances under Other Attacks}

The performances of the proposed work under other attacks like frequency cropping [42], direct current (DC) and high pass filter (HPF) [43] are discussed in this subsection. The definition of DC attack is to pad a certain power on a stego audio, then run the decoding procedure to examine the $B E R$. The definition of HPF attack is to filter all signals whose frequencies are higher than a certain frequency, then run the decoding procedure to examine the BER. The frequency cropping attack 
is to randomly pad signals with certain frequencies on a stego pitch. The presented schemes are conditionally outperformed under the above three attacks.

For frequency cropping attack, if, frequencies of all randomly padded signals are exactly the same with the frequencies of the selected $b_{i} f_{a p} s$, a high BER occurs. Figure 17 presents the experiments of frequency cropping attack. In the setting of the experiment, 50 frequencies are randomly generated to replace the corresponding frequencies of a pitch with different $k$ selected. Another 50 frequencies are generated by normal distribution. In addition, a baseline of the theoretical upper bound is also drawn in the figure.

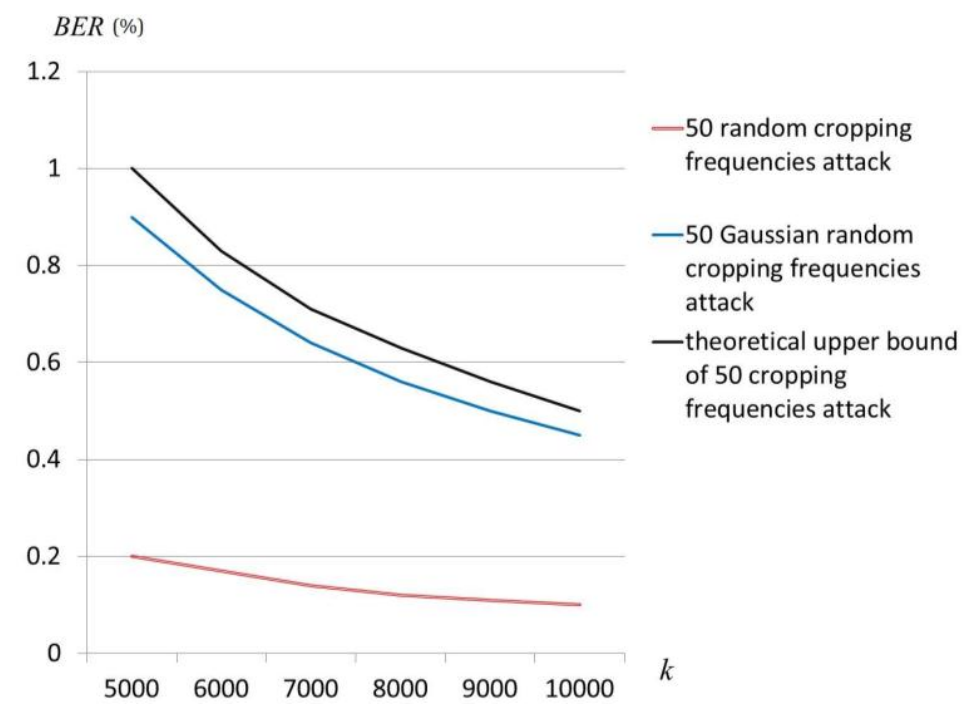

Figure 17. The bit error ratio $(B E R)$ performances of frequency cropping attack.

For DC attack, all sampled values increase after padding a DC signal, and the decoding procedure will fail because the testing condition is the quality of the power of the cover and the stego pitches. However, if the decoding procedure is adequately modified to inspect all amplitudes of $b_{i} f_{a p} s$, it is possible to filter out the DC power and obtain all correct secrets. The inspection could be achieved as follows. Subtract all amplitudes of the selected $b_{i} f_{a p} \mathrm{~s}$ and discover the most appeared value. The most appeared value is the power of DC, and the subtraction of the DC power from the stego pitch will successfully filter out the DC.

For HPF attack, all selected $b_{i} f_{a p}$ s will be filtered out under a certain frequency $f_{H P}$, that is, only those selected $b_{i} f_{a p} \mathrm{~s}$ which are larger than $f_{H P}$ can survive after exploiting HPF on the stego pitch. However, it is a trick to avoid the sabotage of HPF; selecting higher $b_{i} f_{a p}$ will outperform under HPF attack. Table 6 presents the performances under DC and HPF attacks compared with $[17,40,41]$. Two types of the selected $b_{i} f_{a p}$ are used.

Table 6. The BER of the proposed methods and related work. HPF: high pass filter; DC: direct current.

\begin{tabular}{ccc}
\hline Methods/Attacks & HPF (8 kHz) & DC \\
\hline Ours $\left(k=40\right.$, all $b_{i} f_{a p}$ s are larger than 8,000$)$ & $0.0 \%$ & $0.0 \%$ \\
Akhaee et al. [17]: HQ, SQ & $0.0 \%, 0.0 \%$ & $0.0 \%, 0.0 \%$ \\
Wu et al. [40] & $\varnothing$ & $\varnothing$ \\
Chen et al. [41] & $4.2 \%$ & $4.5 \%$ \\
\hline
\end{tabular}

$\varnothing$ : Not mentioned by the corresponding authors.

In recent years, many steganography were proposed with considering the cracking probability [44-51] under a brute force guessing. The threating mode evaluated here is based on 
the intruders knowing algorithms but without the order $R$. In practice, $R$ is a private key and it should be transmitted via a secure channel. If an intruder uses a computer and successfully detects all slight noises between the stego and cover pitch (the standard patterns are also public knowledge), he could dress all enhanced frequencies but without a correct order. This means that he knows which frequency is used to embed " 1 " or " 0 " but he cannot reconstruct the exact bit-stream without $R$. Absolutely, the only thing he can do is to try all combinations of these 1 s and 0 s with $n 1$ s and $i 0$ s where $0 \leq n \leq k$ and $k$ is the number of selected frequencies. Denote the summation of the combinations as $S(n)$. For $n=0$, there are $k$ possibilities of embedded bit-stream because an intruder does not obtain the length of $R$, so that $S(0)=k$.

$$
\begin{aligned}
& \text { For } n=1: S(1)=\frac{1 !}{1 !}+\frac{2 !}{1 ! !}+\ldots+\frac{(k-1) !}{1 !(k-2) !}+\frac{k !}{1 !(k-1) !} \cdot \\
& \text { For } n=2: S(2)=\frac{2 !}{2 !}+\frac{3 !}{2 ! 1 !}+\ldots+\frac{(k-1) !}{2 !(k-3) !}+\frac{k !}{2 !(k-2) !} \cdot \\
& \text { For } n=3: S(3)=\frac{3 !}{3 !}+\frac{4 !}{3 ! 1 !}+\ldots+\frac{(k-1) !}{3 !(k-4) !}+\frac{k !}{3 !(k-3) !} \cdot \\
& \text { For } n=\frac{k}{2}: \\
& S\left(\frac{k}{2}\right)=\frac{\left(\frac{k}{2}\right) !}{\left(\frac{k}{2}\right) !}+\frac{\left(\frac{k}{2}+1\right) !}{\left(\frac{k}{2}\right) ! 1 !}+\frac{\left(\frac{k}{2}+2\right) !}{\left(\frac{k}{2}\right) ! 2 !}+\ldots+\frac{(k-2) !}{\left(\frac{k}{2}\right) !\left(\frac{k}{2}-2\right) !}+\frac{(k-1) !}{\left(\frac{k}{2}\right) !\left(\frac{k}{2}-1\right) !}+\frac{k !}{\left(\frac{k}{2}\right) !\left(\frac{k}{2}\right) !} \\
& \text { For } n=k-3: S(k-3)=\frac{(k-3) !}{(k-3) !}+\frac{(k-2) !}{(k-3) ! 1 !}+\frac{(k-1) !}{(k-3) ! 2 !}+\frac{k !}{(k-3) ! 3 !} . \\
& \text { For } n=k-2: S(k-2)=\frac{(k-2) !}{(k-2) !}+\frac{(k-1) !}{(k-2) ! 1 !}+\frac{k !}{(k-2) ! 2 !} \cdot \\
& \text { For } n=k-1: S(k-1)=\frac{(k-1) !}{(k-1) !}+\frac{k !}{(k-1) ! 1 !} \cdot \\
& \text { For } n=k: S(k)=\frac{k !}{k !} .
\end{aligned}
$$

The above equations reveal a fact that the probability is the same of [S(0), $S(k)],[S(1), S(k-1)]$, $[S(2), S(k-2)], \ldots,\left[S\left(\left\lfloor\frac{k}{2}\right\rfloor-2\right), S\left(\left\lceil\frac{k}{2}\right\rceil+2\right)\right],\left[S\left(\left\lfloor\frac{k}{2}\right\rfloor-1\right), S\left(\left\lceil\frac{k}{2}\right\rceil+1\right)\right],\left[S\left(\left\lfloor\frac{k}{2}\right\rfloor\right), S\left(\left\lceil\frac{k}{2}\right\rceil\right)\right]$. According to the above equations:

$$
S\left(\frac{k}{2}\right)=\sum_{i=0}^{k / 2} \frac{\left(\frac{k}{2}+i\right) !}{(k / 2) ! i !}=\frac{(k+1) !}{\frac{k}{2} ! \frac{k+2}{2} !}
$$

It is the worst case of the successful guessing under brute force. Figure 18 performs $S(k / 2)$ as an exponential function performed and the probability is then equal to $\frac{\frac{k}{2} ! \frac{k}{2} !}{(k+1) !}$.

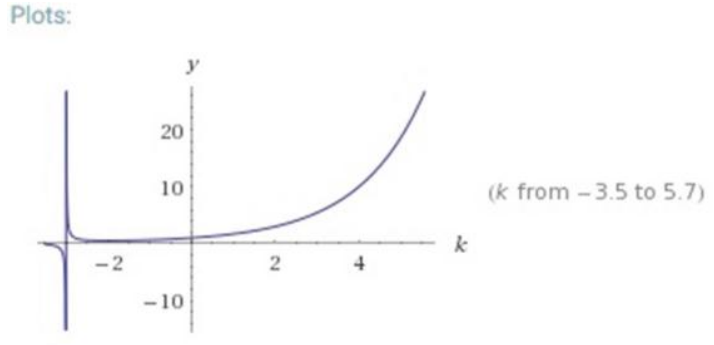

(a)

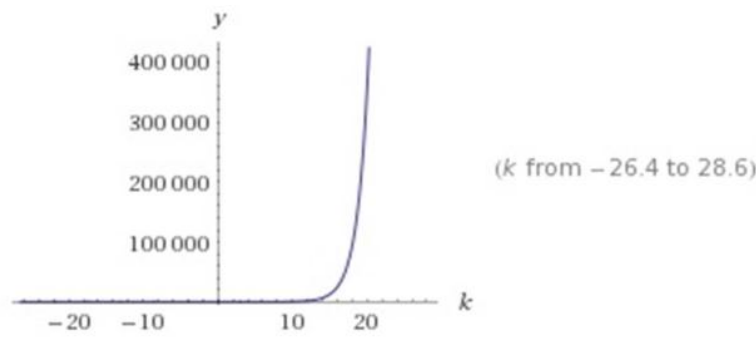

(b)

Figure 18. The distribution of $S(k / 2)$ with: (a) $k$ from -3.4 to 5.7; and (b) $k$ from -26.4 to 28.6.

\subsection{Theoretical Analysis}

This section describes some theoretical analyses of the proposed scheme. Theorem 1 is the theoretical boundary condition of the enhancement. The lower bound is according to the limit of the floating points provided by the software. The upper bound is decided by a user with selected 
number of capacity and expected distortion. Theorem 2 is the theoretical evaluation of the capacity, and it is according to the selected number of hidden secrets. Theorem 3 describes the theoretical time consumption of the proposed scheme. The parameter $n$ is the total sampled data of a pitch and $k$ is the number of secrets. The total computations include DFT, time of data embedding and inverse DFT. Theorem 4 describes the theoretical evaluate of the limit when error bits occur. Because the least bits of the proposed re-quantization are eight bits, this indicates that each datum needs at least eight bits to represent the number. Therefore, if the bit-represent of the enhancement larger than eight, the suffix of the number will disappear when the down-re-quantization (16 to 8 bits) deployed.

Theorem 1. The practical boundary condition of $\sigma$ is $1+10^{-L}<\sigma<1+\frac{1}{k S N R}$ where $L$ is the bit length of the floating point in the corresponding software; $k$ is the number of the selected pitches, and SNR is the set quality of the stego-pitches.

Proof. The value of $\sigma$ is considered here. Ideally, the range of $\sigma$ is 1 to 2 because less noises is better and embedding a signal in a cover pitch that is identical to it is meaningless. A smaller $\sigma$ is preferred because it supports lower distortion and the length of the floating point is limited such that $\lg (\sigma-1)$ cannot exceed the size of the floating point, say $L$, which is defined by the software. Therefore, $\lg (\sigma-1)<L, 1<\sigma<2 \rightarrow 1+10^{-L}<\sigma$ (becasue $0<\sigma-1<1$ ). The practical upper bound is obtained by the setting $\frac{P_{\text {signal }}}{P_{\text {stego }}-P_{\text {signal }}}>$ SNR (signal-to-noise ratio). The denominator $P_{\text {stego }}-P_{\text {signal }}$ causes the embedded noise to stisfy $\frac{1}{k(\sigma-1)}>$ SNR. With fixed $k$ and SNR, the upper bound of $\sigma$ is obtained as $1+\frac{1}{k S N R}$.

Theorem 2. The capacity of the proposed data-hiding scheme is $O(k)$, where $k$ is the number of selected pitches.

Proof. According to Figure 15, the capacity complexity is related to $k$, the number of selected pitches.

Theorem 3. The time complexity of the proposed scheme is $O(n \lg n+k(1+\lg k))$.

Proof. The DFT and inverse DFT cause a temporal bottleneck in the scheme. The best time consumption of DFT and inverse DFT is $O(n \lg n)$, and the time consumed by the proposed scheme, for embedding secrets is $O(k)$. Because $k$ frequencies are selected, the inverse DFT has a time cost of $O(k \lg k)$ rather than $O(n \lg n)$. The total time complexity of the proposed scheme is addressed by the following theory.

Theorem 4. Error bits start to appear if $\lg \sigma>8$.

Proof. The value of $\sigma$ at which the error bit of re-quantization (16 to 8 bits) begins to be caused is of interest. The key point is the bit-size of $\sigma a_{i}$, so $\lg \sigma$ should be smaller than 8 . A theory concerning the production of the error under re-quantization (16 to 8 bits) that corresponds to $\sigma$ is provided.

\section{Conclusions and Future Work}

This work developed a data-hiding scheme that is based on a new cover medium, and synthesized pitches, which are popularly used to demonstrate initial versions of compositions conveniently and at low-cost. This data-hiding scheme relies on the similarity between synthesized pitches and real instrumental pitches to remove concern about the compromising of the hidden of data by audio distortion. To demonstrate the feasibility of the scheme, secret bits are embedded during the generation of simulation of instrumental pitches. Experimental results reveal that more secrets can be hidden without distortion. The proposed method differs from traditional data-hiding schemes in that the data embedding procedure causes insignificant signal distortion. Finally, comparisons of the BER values 
obtained herein and in related work under common attacks reveal that the scheme herein outperforms under some attacks.

The main restriction is that only one pitch can be used at the same time and it is expected that there shall be a more efficient scheme using several pitches at the same time. To achieve more applicability, multiple pitches with multiple instruments used at a single time slot shall be developed. Moreover, according to the progress of technologies of signal processing and the faster computation of computers, exploiting further robust strategies such as spread transform dither modulation is necessary to against attacks.

Acknowledgments: The authors would like to thank the Ministry of Science and Technology, Taiwan, for financially supporting this research under Contract Nos. MOST 105-2221-E-150-063 and MOST 105-2221-E-305-001. The authors would also like to thank Richard Chia-Tung Lee from National Tsing Hua University for his advices on the research works and Ted Knoy for his improvement of English writing (www.chineseowi.idv.tw).

Author Contributions: Hung-Jr. Shiu conceived and designed the experiments; and Chia-Wei Cheng performed the experiments; Hung-Jr. Shiu analyzed the data; Bor-Shing Lin, Chien-Hung Huang and Chin-Laung Lei contributed reagents/materials/analysis tools; and Hung-Jr. Shiu wrote the paper.

Conflicts of Interest: The authors declare no conflict of interest.

\section{References}

1. Katzenbeisser, S.; Petitcolas, F.A.P. Information Hiding Techniques for Steganography and Digital Watermarking; Artech House: Norwood, MA, USA, 2000.

2. Li, B.; He, J.; Huang, J.; Shi, Y.Q. A survey on image steganography and steganalysis. J. Inf. Hiding Multimed. Signal Process. 2011, 2, 142-172.

3. Bender, W.; Morimoto, N.; Liu, A. Techniques for Data Hiding. IBM Syst. J. 1996, 35, 313-336. [CrossRef]

4. Podilchuk, C.I.; Delp, E.J. Digital watermarking: Algorithms and applications. IEEE Signal Process. Mag. 2001, 18, 33-46. [CrossRef]

5. Fridrich, J.; Kodovsky, J. Rich models for steganalysis of digital images. IEEE Trans. Inf. Forensics Secur. 2012, 7, 868-882. [CrossRef]

6. Fridrich, J.; Kodovsky, J.; Holub, V.; Goljan, M. Steganalysis of content-adaptive steganography in spatial domain. In Proceedings of the 13th Information Hiding Conference, Prague, Czech Republic, 19-22 May 2011.

7. Holub, V.; Fridrich, J. Optimizing pixel predictors for steganalysis. In Proceedings of the SPIE, Electronic Imaging, Media Watermarking, Security and Forensics, Burlingame, CA, USA, 22 January 2012.

8. Kodovsky, J.; Fridrich, J. Quantitative steganalysis of triangle strip peeling sequence embedding in JPEG domain. In Proceedings of the ACM Multimedia \& Security Workshop, Rome, Italy, 9-10 September 2010.

9. Kodovsky, J.; Fridrich, J. Quantitative structural steganalysis of Jsteg. IEEE Trans. Inf. Forensics Secur. 2010, 5, 681-693. [CrossRef]

10. Kodovsky, J.; Fridrich, J. Steganalysis in high dimensions: Fusing classifiers built on random subspaces. In Proceedings of the SPIE, Electronic Imaging, Media Watermarking, Security and Forensics, San Francisco, CA, USA, 23 January 2011.

11. Kodovsky, J.; Fridrich, J. Steganalysis of JPEG images using rich models. In Proceedings of the SPIE, Electronic Imaging, Media Watermarking, Security and Forensics, Burlingame, CA, USA, 22 January 2012.

12. Kodovsky, J.; Fridrich, J.; Holub, V. On dangers of overtraining steganography to incomplete cover model. In Proceedings of the ACM Multimedia \& Security Workshop, Niagara Falls, NY, USA, 29-30 September 2011.

13. Kodovsky, J.; Fridrich, J.; Holub, V. Ensemble classifiers for steganalysis of digital media. IEEE Trans. Inf. Forensics Secur. 2012, 7, 432-444. [CrossRef]

14. Kodovsky, J.; Pevny, T.; Fridrich, J. Modern steganalysis can detect YASS. In Proceedings of the SPIE, Electronic Imaging, Media Forensics and Security, San Jose, CA, USA, 17 January 2010.

15. Pevny, T.; Bas, P.; Fridrich, J. Steganalysis by subtractive pixel adjacency matrix. IEEE Trans. Inf. Forensics Secur. 2010, 5, 215-224. [CrossRef]

16. Pevny, T.; Fridrich, J.; Ker, A.D. From blind to quantitative steganalysis. IEEE Trans. Inf. Forensics Secur. 2011, 7, 445-454. [CrossRef] 
17. Akhaee, M.A.; Saberian, M.J.; Feizi, S.; Marvasti, F. Robust audio data hiding using correlated quantization with histogram based detector. IEEE Trans. Multimed. 2009, 11, 834-842. [CrossRef]

18. Atoum, M.S.; Al-Rababah, O.A.; Al-Attili, A.I. New technique for hiding data into audio file. Int. J. Comput. Sci. Netw. Secur. 2011, 11, 173-177.

19. Huang, X.; Abe, Y.; Echizen, I. Capacity adaptive synchronized acoustic steganography scheme. J. Inf. Hiding Multimed. Signal Process. 2010, 1, 72-90.

20. Swanson, M.D.; Zhu, B.; Tewfik, A.H.; Boney, L. Robust watermarking using perceptual masking. Signal Process. 1998, 66, 337-355. [CrossRef]

21. Tilki, J.F.; Beex, A.A. Encoding a hidden digital signature onto an audio signal using psychoacoustic masking. In Proceedings of the 1996 7th International Conference on Signal Processing Applications and Technology, Boston, MA, USA, 7-10 October 1996.

22. Valizadeh, A.; Wang, Z.J. An improved multiplicative spread spectrum embedding scheme for data hiding. IEEE Trans. Inf. Forensics Secur. 2012, 7, 1127-1143. [CrossRef]

23. Xiang, Y.; Natgunanathan, I.; Peng, D.; Zhou, W.; Yu, S. A dual channel time spread echo method for audio watermarking. IEEE Trans. Inf. Forensics Secur. 2012, 7, 383-392. [CrossRef]

24. Yamamoto, K.; Iwakiri, M. Real time audio watermarking based on characteristics of PCM in digital instrument. J. Inf. Hiding Multimed. Signal Process. 2010, 1, 59-71.

25. Desoky, A. NORMALS: Normal linguistic steganography methodology. J. Inf. Hiding Multimed. Signal Process. 2010, 1, 145-171.

26. Grosvald, M.; Orgun, C.O. Free from the cover text: A human-generated natural language approach to text-based steganography. J. Inf. Hiding Multimed. Signal Process. 2011, 2, 133-141.

27. Kermanidis, K.L. Capacity-rich knowledge-poor linguistic steganography. J. Inf. Hiding Multimed. Signal Process. 2011, 2, 247-258.

28. Cayre, F.; Macq, B. Data hiding on 3D triangle meshes. IEEE Trans. Signal Process. 2003, 51, 939-949. [CrossRef]

29. Chao, M.W.; Lin, C.H.; Yu, C.W.; Lee, T.Y. A high capacity 3D steganography algorithm. IEEE Trans. Vis. Comput. Graph. 2008, 15, 274-284. [CrossRef] [PubMed]

30. Ritchey, P.C.; Rego, V.J. A context sensitive tiling system for information hiding. J. Inf. Hiding Multimed. Signal Process. 2012, 3, 212-226.

31. Wang, C.M.; Cheng, Y.M. An Efficient Information Hiding Algorithm for Polygon Models; Eurographics: Dublin, Ireland, 19 August 2005.

32. Cheng, Y.M.; Wang, C.M. A high capacity steganographic approach for 3D polygonal meshes. Visual Comput. 2006, 22, 845-855. [CrossRef]

33. Aguilar, J.R.; Salinas, R. New trend in sound synthesis and automatic tuning of electronic musical instruments. Revista Fac. Ing. 2003, 11, 17-23. [CrossRef]

34. Fletcher, H.; Blackham, E.D.; Stratton, R. Quality of piano tones. J. Acoust. Soc. Am. 1962, 34, 749-761. [CrossRef]

35. Casey, M.A. Understanding musical sound with forward models and physical models. Connect. Sci. 1994, 6, 355-371. [CrossRef]

36. Chaigne, A.; Askenfelt, A. Numerical simulations of piano strings: A physical model for a struck string using finite difference methods. J. Acoust. Soc. Am. 1993, 95, 1112-1118. [CrossRef]

37. Smith, J.O. A history of ideas leading to virtual acoustic musical instruments. In Proceedings of the IEEE Workshop on Applications of Signal Processing to Audio and Acoustics, New Paltz, NY, USA, 16 October 2005; pp. 299-306.

38. Lee, R.C.T.; Chiu, M.C.; Lin, J.S. Communications Engineering: Essentials for Computer Scientists and Electrical Engineers; Wiley-IEEE Press: Piscataway, NJ, USA, 2007.

39. Lee, R.C.T.; Tseng, S.S.; Tsai, Y.T. Introduction to the Design and Analysis of Algorithms; McGrawHill: New York, NY, USA, 2007; pp. 223-224.

40. Wu, S.; Huang, J.; Huang, D.; Shi, Y.Q. Efficiently self-synchronized audio watermarking for assured audio data transmission. IEEE Trans. Broadcast. 2005, 51, 69-76. [CrossRef]

41. Chen, O.T.C.; Wu, W.C. Highly robust, secure, and perceptual-quality echo hiding scheme. IEEE Trans. Audio Speech Lang. Process. 2008, 16, 629-638. [CrossRef] 
42. Huang, Y.H.; Chang, C.C.; Wu, C.Y. A DNA-based data hiding technique with low modification rates. Multimed. Tools Appl. 2014, 70, 1439-1451. [CrossRef]

43. Shiu, H.J.; Tang, S.Y.; Huang, C.H.; Lee, R.C.T.; Lei, C.L. A reversible acoustic data hiding method based on analog modulation. Inf. Sci. 2014, 273, 233-246. [CrossRef]

44. Khalifa, A.; Elhadad, A.; Hamad, S. Secure blind data hiding into pseudo DNA sequences using playfair ciphering and generic complementary substitution. Appl. Math. Inf. Sci. 2016, 10, 1483-1492. [CrossRef]

45. Marwan, S.; Shawish, A.; Nagaty, K. DNA-based cryptographic methods for data hiding in DNA media. Biosystems 2016, 150, 110-118. [CrossRef] [PubMed]

46. Hafeez, I.; Khan, A.; Qadir, A. DNA-LCEB: A high-capacity and mutation-resistant DNA data-hiding approach by employing encryption, error correcting codes, and hybrid twofold and fourfold codon-based strategy for synonymous substitution in amino acids. Med. Biol. Eng. Comput. 2014, 52, 945-961. [CrossRef] [PubMed]

47. Tulpan, D.; Regoui, C.; Durand, G.; Belliveau, L.; Leger, S. HyDEn: A hybrid steganocryptographic approach for data encryption using randomized error-correcting DNA codes. BioMed Res. Int. 2013, 2013, 634832. [CrossRef] [PubMed]

48. Bhattacharyya, D.; Bandyopadhyay, S.K. Hiding secret data in DNA sequence. Available online: http: / / www.ijser.org/researchpaper/Hiding-Secret-Data-in-DNA-Sequence.pdf (accessed on 17 June 2017).

49. Kong, W.; Bhattacharyya, D.; Bandyopadhyay, S.K. Embedding data in DNA sequence for security. Int. J. Reliab. Inf. Assur. 2013, 1, 1-6.

50. Abbasy, M.R.; Nikfard, P.; Ordi, A. DNA based data hiding algorithm. Int. J. New Comput. Archit. Appl. 2012, 2, 183-192.

51. Shiu, H.J.; Ng, K.L.; Fang, J.F.; Lee, R.C.T.; Huang, C.H. Data hiding methods based upon DNA sequences. Inf. Sci. 2010, 180, 2196-2208. [CrossRef]

(C) 2017 by the authors. Licensee MDPI, Basel, Switzerland. This article is an open access article distributed under the terms and conditions of the Creative Commons Attribution (CC BY) license (http:/ / creativecommons.org/licenses/by/4.0/). 